\title{
The effect of daily change of sterile urinary bag on bacteriuria and catheter-associated urinary tract infections
}

\author{
Masoud Hafizi $^{1}{ }^{\circledR}$, Mohammad Rajaei-Isfahani $^{*{ }^{*}}{ }^{\circledR}$ Kobra Noorian $^{3}$ \\ ${ }^{1}$ Department of Infectious Diseases, Shahrekord University of Medical Sciences, Shahrekord, Iran \\ ${ }^{2}$ Department of Urology, Shahrekord University of Medical Sciences, Shahrekord, Iran \\ ${ }^{3}$ Lecturer, Shahrekord University of Medical Sciences, Shahrekord, Iran
}

\section{A R T I C L E I N F O}

Article Type:

Original

\section{Article History:}

Received: 13 February 2018

Accepted: 20 June 2018

Published online: 15 July 2018

Keywords:

Bacteriuria

Urinary tract infection

Urethral catheterization

\begin{abstract}
A B S T R A C T
Introduction: One of the major healthcare measures in hospitals, is reducing the risk of infections. Since catheter-associated urinary tract infections (CA-UTIs) occur in $80 \%$ of cases, the prevention of these infections plays an important role in reducing infections related to the treatment care. Objectives: The present study aims to determine the effect of daily change of sterile urinary bag on the incidence of bacteriuria and CA-UTIs.

Patients and Methods: This quasi-experimental study include all patients referred to the neurology, urology and surgery wards, as well as the ICU and CCU of Hajar and Kashani hospitals, Shahrekord, Iran. A total of 200 patients aged 18-75 years that required insertion of the urinary Foley catheter were divided into two groups: catheterization with the usual method (control group) and catheterization with daily change of sterile urinary bag (case group). In each of the two groups, immediately after insertion and during the removal of urethral catheter, the urine sample was collected using sterile method and was sent to the laboratory to conduct examinations for bacteriuria. The demographic information was prepared and collected using the researcher made questionnaire.

Results: In this study, the average age of the patients was $49.81 \pm 15.11$. The rate of CA-UTI was more in the control group than the case group ( $29 \%$ as compared with $16 \%)(P=0.04)$. The average age of patients with infection due to urethral catheterization was found to be higher $(55.4 \pm 14.52$ versus $48.4 \pm 14.92)(P<0.001)$.

Conclusion: While a preference for closed drainage system existed, however in the present study, it was found that with daily change of sterile urinary bag even with opening of system, the rate of CA-UTI was reduced.
\end{abstract}

Implication for health policy/practice/research/medical education:

According to the findings of the present study, in short-term catheterization, replacing sterile urine bag was more effective in decreasing bacteriuria and urinary tract infections (UTIs) as compared with the conventional method of urinary catheterization. Please cite this paper as: Hafizi M, Rajaei-Isfahani M, Noorian K. The effect of daily change of sterile urinary bag on bacteriuria and catheter-associated urinary tract infections. J Renal Inj Prev. 2018;7(4):240-245. DOI: 10.15171/jrip.2018.55.

\section{Introduction}

At present, hospital acquired infections are one the main causes of the increasing rate of complications and mortality in hospitalized patients. These infections are threat to the health condition of the individuals and cause a heavy economic burden on health care systems, individuals, and insurance organizations (1). Studies have shown that the urinary system accounts for approximately $40 \%$ of all hospital-acquired infections. Infections of the urinary tract are the most common types of healthcareassociated infection in medical-surgical units, critical care units, and rehabilitation wards and approximately $80 \%$ are associated with the use of an indwelling urinary catheter $(2,3)$.

The prevalence of urethral catheterization is high, about $15 \%$ to $25 \%$ of patients hospitalized in the emergency department have urinary urethral catheter and more than half of them are affected by bacteriuria after five days of hospitalization; thus, it is present as a consequence of interventional procedures (4). For each day of urethral catheterization, the rate of acquiring bacteriuria is approximately $1 \%-3 \%$ in healthy individuals and $15 \%$ in elderly hospitalized patients (4). This rate, after 7 to 14 days of catheterization, increases between $35 \%$ 
and $75 \%$, respectively and the bacteriuria is likely to be asymptomatic. About $20 \%$ of patients with bacteriuria are diagnosed with symptomatic urinary tract infections (3). On the other hand, the urinary tract infections occur more in lower urinary tract which has high rate of colonized bacteria. The insertion of urethral catheter could help to reduce this condition and causes bacteriuria. In creation of this problem, the biofilm formation plays an important role.

Biofilm is a polysaccharide that is formed in the urinary bag on the urethral catheter, in openings and urethral catheter balloon. It prepares the condition for bacterial growth and infection $(3,5)$.

The responsible factors of biofilm (bacterium, fungus, and protozoan) play an important role in causing these infections. About $60 \%$ of urinary tract infections occur in the presence of biofilm (6). With biofilm formation, the bacteria can develop resistance against anti-microbial factors and immunity system, and would be able to keep the proper physical and chemical conditions for growing. The essential time for biofilm formation on instruments depends on the type of micro-organism and instrumental material.

However, on average, 24 hours is required to attain the thickness of biofilm (7). With biofilm formation, bacteria can develop resistant against antibacterial materials and this process leads to chronic or recurrent infections that is difficult to treat. In the absence of treatment, these infections can lead to pyelonephritis, bacteremia, chronic bacterial prostatitis, bacterial vaginitis, chronic pyelonephritis, bladder cancer, and in some cases, to death (7-9).

Several strategies have been suggested with the aim of reducing the rate of catheter-associated urinary tract infection (CA-UTI), including training of the patients and their health providers and personnel to wash their hands, restricting the use of urethral catheter only for emergency cases, using aseptic techniques for urethral catheterization, using antibiotics only for special cases, keeping a closed (drainage) system, repeated emptying of the urinary bag in order to prevent urinary reflux, minimizing the duration of catheterization, and using catheter coated with anti-biotics or antiseptics (10).

Furthermore, several studies have been carried out on the application of strategies to reduce CA-UTI and bacteriuria in patients. Koskeroghlo et al (11) in their study, showed that using antiseptics in the urinary tract was not effective in reducing the rate of acquiring bacteriuria. Also, Schumm et al (12) demonstrated that none of the bladder washing solutions (acetic acid, neomycin- polymyxin, and sodium chloride $0.9 \%$ ) could serve as a suitable antibacterial agent to prevent catheterization-related bacteriuria. In another study, it was shown that the application of betadine on urinary tract once and twice a day was associated with prevention of CI-UTIs in men and women, respectively $(13,14)$.

\section{Objectives}

This study was conducted with the aim of determining the effect of daily change of sterile urinary bag on incidence of bacteriuria and CA-UTIs.

\section{Patients and Methods \\ Study population}

This quasi-experimental study included all patients referred to the neurology, urology, and surgery wards, as well as the ICU and CCU of Hajar and Kashani hospitals, Shahrekord, Iran. The inclusion criteria were staying in the studied wards of the two hospital, needing urinary catheter, and being 18-75 years. The exclusion criteria were positive urinary culture at baseline, having undergone any surgery on the urinary tract, and taking antibiotics.

The sample size was estimated to be 200 persons. Data collection was conducted using a researcher-developed questionnaire that consisted of items on demographic characteristics and underlying information on the wards of hospitalization, catheterization indications, the type of catheter, and the technique of catheterization. This questionnaire was developed according to the relevant textbooks and articles, and the viewpoints of the faculty members of the departments of urology and infectious diseases. The viewpoints of 10 faculty members of the departments of urology and infectious diseases were used to validate the questionnaire according to content validity. The sampling was done based on the objective and samples specification in the two groups, randomly. About 200 qualified cases were considered for the study and were divided into two groups. In the case group of 50 female and 50 male, the silicon urethral catheter was established using aseptic method. Then, after filling each urinary bag, it was intermittently emptied and changed daily. In the control group (50 female, 50 male), also the silicon urethral catheter was established using routine and aseptic methods, and the urinary bag was emptied after each filling.

In all two groups of the study, the urinary catheter was inserted using the sterile technique. Simultaneously, urinary samples were collected from the urinary catheters using 10-cc sterile syringe and sent to the laboratory in less than 30 minutes to conduct examinations for bacteriuria. In both groups, immediately after removing the urinary catheter, the urinary samples were collected again from the catheters by aseptic technique using a 10-cc sterile syringe and sent to the laboratory under similar conditions. The urinary samples were examined for bacterial infection (over $1000 \mathrm{CFU} / \mathrm{mL}$ ) (15).

To conduct urinary tests, sterilized test tubes with cotton lids, blood agar, McConkey culture medium, optical microscope, and incubator were used. All urinary tests were conducted by a single person and in a laboratory.

\section{Ethical issues}

The study protocol was in accordance with the 
Declaration of Helsinki. Ethical permission was obtained from the ethical committee of Shahrekord University of Medical Science. Before collecting the urinary samples, the research purposes were explained to the patients, and then they provided informed consent to participate in the study.

\section{Statistical analysis}

Data analysis was conducted by descriptive (frequency, relative frequency, mean, and standard deviation) and analytical statistics by SPSS. The Kolmogorov-Smirnov statistical test showed that the data had normal distribution. Thus, the parametric (chi-square and independent $t$ test) tests were used to analyze the data. Additionally, $P<0.05$ was considered statistically significant.

\section{Results}

A total of 200 patients were enrolled in this study. The mean age of the patients was $49.81 \pm 15.11$ years. The duration of catheterization ranged from 3 to 10 days. About 36 patients were hospitalized in the neurology ward, 44 in the urology ward, 40 in the ICU, 20 in the CCU, and 60 in the surgical ward. There were no significant differences in age, gender, and catheter survival among the studied groups $(P<0.05)$.

Table 1 shows that the most prevalent causative organism of CA-UTIs was Escherichia coli (25 patients).

Comparison of the uninfected and infected groups by chisquare test showed a significant difference of urinary tract infection in the two methods of urethral catheterization $(P=0.033)$. The urinary infection rate was reported as $16 \%$ in the case group and $29 \%$ in the control group.

The average age of patients with infection due to the urethral catheterization was reported as $55.4 \pm 14.52$ years, and for patients without infection as $48.4 \pm 14.92$ years. The independent $t$ test demonstrated a significant difference between age and urinary tract infection rate $(P<0.001)$. This finding shows that infection rate is more prevalent among older people.

Furthermore, the independent $t$ test demonstrated a significant difference in duration time of urethral catheter and CA-UTIs $(P<0.001)$.

Moreover, there was no significant difference in CA-UTIs in the two genders $(P>0.05$; chi-square test). Although the urinary infection incidence was more in women (female) than in men (male) (31\% versus 14\%), however, it was not statistically significant. There was a significant difference in CA-UTIs among the males of the two groups $(P=0.4$; chi-square test). However, the females in the two groups had no significant difference in CA-UTIs $(P=0.13$; chisquare test). Hence, only the males in the two groups had significantly different incidence rate of CA-UTIs.

The most infection rate was observed in ICU unit (47.2\%), while the least infection rate was observed in CCU (20\%). $\chi^{2}$ tests showed that the effect of hospitalization unit was significant in CA-UTIs $(P<0.001)$. The incidence rate of different complication in the control group was $41 \%$ and in the case group was $33 \%$.

Additionally, dysuria was observed more than other complications (37\%), and it was 19\% in the control group and $17 \%$ in the case group (Table 2).

\section{Discussion}

Control measures to decrease healthcare-associated infections are a priority in hospitals of developed countries. While, catheterization-related UTIs are one of the most common hospital acquired infections and occur in $80 \%$ of cases following catheterization, preventing such infections plays an important role in decreasing healthcare-associated infections. Therefore, the present study was conducted to compare the efficacy of urinary catheterization using daily urine drainage bags and catheterization using daily replacement of urine bag on bacteriuria and catheter-related UTIs.

Based on the findings of the present study, urine bag replacement instead of its drainage was effective in decreasing bacteriuria and UTIs following urinary catheterization as compared with the conventional method of urine drainage bags. Our finding represents the effectiveness of urine bag replacement in decreasing biofilm development, because biofilms are polysaccharides that protect bacteria.

In this regard, no similar study has been conducted. However, the adoption of strategies recommended by the disease control and prevention (CDC) to prevent or decrease the catheterization-related UTIs has been frequently studied. It is not possible to reach a definite conclusion due to inconsistency in the available findings. For example, Makuta et al (10) investigated the efficacy of antibacterial catheters. To achieve this purpose, they compared the use of silver and nitrofurazone-impregnated catheters and conventional catheters to decrease catheterrelated UTIs. The findings showed that the use of silver and nitrofurazone-impregnated catheters was ineffective

Table 1. The distribution of urinary tract infection (UTI) in terms of organism type and treatment methods

\begin{tabular}{|c|c|c|c|c|c|c|c|}
\hline \multirow{3}{*}{ Groups } & \multicolumn{6}{|l|}{ Infection } & \multirow{3}{*}{ Total } \\
\hline & \multicolumn{2}{|l|}{ Uninfected } & \multicolumn{4}{|c|}{ Infected } & \\
\hline & Lack of infection & Sterile pyuria & Mixed & E. coli & Pseudomonas & Others & \\
\hline Intermittent urinary bag drainage (control group) & 62 & 9 & 7 & 17 & 3 & 2 & 100 \\
\hline $\begin{array}{l}\text { The sterile bag change (the intermittent urinary bag } \\
\text { and daily change of the urinary bag) (case group) }\end{array}$ & 76 & 8 & 5 & 8 & 1 & 2 & 100 \\
\hline Total & 138 & 17 & 12 & 25 & 4 & 4 & 200 \\
\hline
\end{tabular}


Table 2. The frequency of the created problems in the two groups as a consequence of urethral catheterization

\begin{tabular}{|c|c|c|c|c|c|c|c|c|c|c|}
\hline \multirow{2}{*}{ Groups } & & \multicolumn{8}{|c|}{ Complications } & \multirow[b]{2}{*}{ Total } \\
\hline & & Fever & Flank pain & $\begin{array}{c}\text { Catheter } \\
\text { obstruction }\end{array}$ & $\begin{array}{c}\text { Urinary } \\
\text { incontinence }\end{array}$ & Dysuria & Hematuria & Wound & $\begin{array}{l}\text { Urinary tract } \\
\text { obstruction }\end{array}$ & \\
\hline \multirow{2}{*}{ Control group } & No. & 5 & 6 & 3 & 0 & 19 & 6 & 1 & 1 & 41 \\
\hline & $\%$ & 2.5 & 3 & 1.5 & 0 & 9.5 & 3 & 1 & 1 & 20.5 \\
\hline \multirow{2}{*}{ Case group ${ }^{b}$} & No. & 6 & 3 & 0 & 0 & 18 & 6 & 0 & 0 & 33 \\
\hline & $\%$ & 3 & 1.5 & 0 & 0 & 9 & 3 & 0 & 0 & 16.5 \\
\hline \multirow{2}{*}{ Total } & No. & 11 & 9 & 3 & 0 & 37 & 12 & 1 & 1 & 74 \\
\hline & $\%$ & 5.5 & 4.5 & 1.5 & 0 & 18.5 & 6 & .5 & .5 & 37 \\
\hline
\end{tabular}

antermittent urinary bag drainage (usual method).

${ }^{b}$ The sterile bag changes (the intermittent urinary bag and daily change of the urinary bag).

in decreasing such infections as compared with the conventional catheters without any benefits. Their finding did not support the use of antibacterial catheters instead of conventional methods. Therefore, healthcare providers are recommended to adopt simple strategies to prevent catheter-related UTIs and decrease the need for catheterization. In this regard, the present study also showed that the method of replacing sterile urine bag was effective in decreasing UTIs as compared with the urine drainage bags.

Pickard et al (16) studied the effects of different types of urinary catheters in decreasing symptomatic UTIs in hospitalized adults. They compared nitrofurazoneimpregnated silicone catheters and silver alloyimpregnated hydrogel latex catheters with PTFE (polytetrafluoroethylene) coated latex-impregnated catheters. According to the findings, using the nitrofurazone-impregnated catheters was not effective in decreasing UTIs but was more economical than the PTFE-impregnated latex catheters.

In addition, the use of the silver alloy-impregnated catheters was neither more effective in decreasing UTIs nor more economical than the PTFE-impregnated catheters. Due to discrepancy in the results of the study by Pickard et al (11), our findings can be related to the duration of catheterization. Moreover, a systematic review by Schumm and (12) to comparatively investigate the effects of silver alloy-impregnated catheters and silver oxide-impregnated method to decrease catheter-related UTIs in short-term catheterization, it was shown that the silver alloy-impregnated catheters were more effective. This review also reported that in short-term (less than oneweek) catheterization, antibacterial agent-impregnated catheters were effective in decreasing bacteriuria, but in long-term catheterization, the results were tentative (12). However, a study by Saint et al (13) demonstrated that the use of silver alloy-impregnated catheters and silver acidimpregnated catheters did not show significant difference in terms of prevention of UTIs.

A review to investigate the effects of antibacterial catheters to decrease bacteriuria and funguria showed that these catheters were effective in decreasing bacteriuria and funguria, but did not report their effects on UTIs rate (14). These findings are in line with the systematic review conducted by Johnson et al (15) who reported that antibacterial agent-impregnated catheters were effective in decreasing UTIs. In the present study, in the shortterm catheterization, the replacement of sterile urine bag caused a decrease in the rates of bacteriuria and UTIs. Accordingly, a study by Koskeroglu et al (11) showed that applying disinfectant agents on urinary meatus was ineffective in decreasing bacteriuria rate. Also, Fukunaga et al (17), in their study, showed that applying iodine povidone on the dressing site of subclavian venous catheter caused a decrease in infection in the catheter site. In contrast, Bastable et al (18) reported that washing was ineffective in decreasing bacteriuria rate.

In the present study, the incidence rate of UTIs following urinary catheterization was $45 \%$. In the study of Haley et al (19), this rate was 10\%-20\%. Edmond et al (20) reported that UTIs represent $40 \%$ of hospital acquired infections, while $80 \%$ of which are developed after catheterization. The duration of catheterization is an important risk factor for the development of catheter-related bacteriuria $(21,22)$. For each 24 -hour of catheterization, the likelihood of bacteriuria development increases by 1-3 and 15\% in healthy people and older patients, which increase to $35 \%$ and $75 \%$, respectively after $7-14$ days of catheterization (3).

In the present study, the duration of catheterization was directly associated with the development of bacteriuria. As reported by Tambyah et al (23), having urinary catheter for more than six days is a modifiable and known risk factor for developing UTIs. Samimi et al (24) also reported bacterial colonization of bladder after 17 days in both groups. Similarly, Al-Hazmi (25), in a study, showed that reduction in the length of hospital stay and duration of catheterization was effective in decreasing the rate of catheter-related UTIs.

In the present study, the highest rate of catheter-related UTIs was reported in ICU, which is in line with the findings of Chen et al (26). Wu et al (21) reported that E. coli $(80 \%)$ is the most common cause of community-acquired infections, followed by Staphylococcus saprophyticus (10\%- 
15\%), Klebsiella, Enterobacter, and Proteus. Consistently, E. coli was the most common cause of UTIs in the present study. This finding is in agreement with those of previous studies (27-29). In the present study, the most frequent complaint following catheterization, by all methods, was dysuria. Urinary catheter can cause dysuria through the development of inflammation and infection in the bladder (30).

\section{Conclusion}

Based on the findings of the present study, in short-term catheterization, replacing sterile urine bag was more effective in decreasing bacteriuria and UTIs as compared with the conventional method of urinary catheterization, that is, urine bag drainage. Decreasing the inflammation status in the body and provocation of oxidative stress would be accompanied by this modality. It should also be noted that prolonged UTI may gradually involve the upper urinary system.

\section{Limitations of the study}

The limitations of the study were the small sample size and short time follow-up. Therefore, further studies with large sample size and longer duration of follow-up of patients are required.

\section{Acknowledgments}

The authors are grateful to the Research and Technology Deputy of the Shahrekord University of Medical Sciences and all who helped in conducting this study.

\section{Authors' contribution}

$\mathrm{MH}$ conducted the research. KN gathered the data. KN analyzed the data. MRI and MH prepared the primary draft. KN edited the manuscript. KN prepared the final paper. All authors read and approved the final manuscript.

\section{Conflicts of interest}

The authors declare no conflict of interest.

\section{Ethical considerations}

Ethical issues (including plagiarism, data fabrication, double publication) have been completely observed by the authors.

\section{Funding/Support}

The Shahrekord University of Medical Sciences financially supported this study.

\section{References}

1. Hooton TM, Bradley SF, Cardenas DD, Colgan R, Geerlings SE, Rice JC, et al. Diagnosis, prevention, and treatment of catheter-associated urinary tract infection in adults: 2009 International Clinical Practice Guidelines from the Infectious Diseases Society of America. Clin Infect Dis. 2010;50:625-63.

2. Lam TB, Omar MI, Fisher E, Gillies K, MacLennan S.
Types of indwelling urethral catheters for short-term catheterization in hospitalized adults. Cochrane Database Syst Rev. 2014;(9):CD004013. doi: 10.1002/14651858. CD004013.pub4.

3. Parker D, Callan L, Harwood J, Thompson D, Wilde M, Gray M. Nursing interventions to reduce the risk of catheter-associated urinary tract infection. Part 1: Catheter selection. J Wound Ostomy Continence Nurs. 2009; 36:2334. doi: 10.1097/01.WON.0000345173.05376.3e.

4. Parida S, Mishra S. Urinary tract infections in the critical care unit: A brief review. Indian J Crit Care Med. 2013; 17:370-4. doi: 10.4103/0972-5229.123451.

5. Parry MF, Grant B, Sestovic M. Successful reduction in catheter-associated urinary tract infections: focus on nurse-directed catheter removal. Am J Infect Control. 2013;41:1178-81. doi: 10.1016/j.ajic.2013.03.296.

6. Ponnusamy P, Natarajan V, Sevanan M. In vitro biofilm formation by uropathogenic Escherichia coli and their antimicrobial susceptibility pattern. Asian Pacific J Trop Med 2012;5:210-213.

7. Waites KB, Canupp KC, Roper JF, Camp SM, Chen Y. Evaluation of 3 method of bladder irrigation to treat bacteriuria in persons with neurogenic bladder. J Spinal Cord Med. 2006; 29:217-26.

8. Basami K, Mahdavi Z, Nikravan Mofrad M, Kohestani $\mathrm{H}$, Baghcheghi N. Effects of disinfecting meatus and urinary catheter with $10 \%$ Povidone Iodine ointment on incidence rate of bacteriuria in hospitalized male patients in neurologic wards. J Arak Univ Med Sci. 2008; 11:10-18.

9. Maki DG, Tambyah PA. Engineering out the risk of infection with urinary Catheters. Emerg Infect Dis. 2001; 7:342-7. doi:10.3201/eid0702.700342.

10. Makuta G, Chrysafis M, Lam T. Measuring the efficacy of antimicrobial catheters. Nurs Times. 2013;109:16, 18-9.

11. Koskeroglu N, Durmaz G, Bahar M, Kural M, Yelken B. The role of meatal disinfection in preventing catheterrelated bacteriuria in an intensive care unit: a pilot study in Turkey. J Hosp Infect. 2004;56:236-8. doi: 10.1016/j. jhin.2003.12.017.

12. Schumm K, Lam TB. Types of urethral catheters for management of short-term voiding problems in hospitalized adults: a short version Cochrane review. Neurourol Urodyn. 2008;27:738-46. doi: 10.1002/nau.20645.

13. Saint S, Veenstra DL, Sullivan SD, Chenoweth C, Fendrick AM. The potential clinical and economic benefits of silver alloy urinary catheters in preventing urinary tract infection. Arch Intern Med. 2000; 160:2670-5. doi: 10.1001/ archinte.160.17.2670.

14. Drekonja DM, Kuskowski MA, Wilt TJ, Johnson JR. Antimicrobial urinary catheters: a systematic review. Expert Rev Med Devices. 2008;5:495-506. doi: 10.1586/17434440.5.4.495.

15. Johnson JR, Kuskowski MA, Wilt TJ. Systematic review: antimicrobial urinary catheters to prevent catheterassociated urinary tract infection in hospitalized patients. Ann Intern Med. 2006; 144:116-26.

16. Pickard R, Lam T, Maclennan G, Starr K, Kilonzo M, McPherson G, et al. Types of urethral catheter for reducing symptomatic urinary tract infections in hospitalized adults requiring short-term catheterization: multicenter randomized controlled trial and economic evaluation of antimicrobial- and antiseptic-impregnated urethral catheters (the CATHETER trial). Health Technol Assess. 
2012;16:1-197. doi: 10.3310/hta16470.

17. Fukunaga A, Naritaka H, Fukaya R, Tabuse M, Nakamura T. Our method of Povidone-Iodine ointment and gauze dressings reduced catheter-related infection in serious Cases. Dermatology. 2006;212:47-52. doi: 10.1159/000089199.

18. Bastable JR, Peel RN, Birch DM, Richards B. Continuous Irrigation of the Bladder after Prostatectomy: Its Effect on Post-Prostatectomy Infection. Br J Urol. 1977; 49:689-93.

19. Haley RW, Hooton TM, Culver DH, Stanley RC, Emori TG, Hardison CD, et al. Nosocomial infections in U.S. hospitals, 1975-1976: estimated frequency by selected characteristics of patients. Am J Med. 1981;70:947-59.

20. Edmond MB, Wallace SE, McClish DK, Pfaller MA, Jones RN, Wenzel RP. Nosocomial bloodstream infections in United States hospitals: a three-year analysis. Clin Infect Dis. 1999;29:239-44. doi: 10.1086/520192.

21. Wu CJ, Lee HC, Lee NY, Shih HI, Ko NY, Wang LR, et al. Predominance of Gram-negative bacilli and increasing antimicrobial resistance in nosocomial bloodstream infections at a university hospital in southern Taiwan, 1996-2003. J Microbiol Immunol Infect. 2006; 39:135-43.

22. Janzen J, Buurman BM, Spanjaard L, de Reijke TM, Goossens A, Geerlings SE. Reduction of unnecessary use of indwelling urinary catheters. BMJ Qual Saf. 2013; 22:984-8. doi: 10.1136/bmjqs-2013-001908.

23. Tambyah PA, Halvorson K, Maki DG. A prospective study of the pathogenesis of catheter-associated urinary tract infection. Mayo Clin Proc. 1999;74:131-6. doi:
10.4065/74.2.131.

24. Samimi G, Ezati Q, Saroukhi M, Mosallaei S, Mehrabi Y. Comparison of irrigation of the Bladder with chlorohexidine and sodium chloride to prevention of bacteriuria. Nurs Mid J. 2011:20:23-27.

25. Al-Hazmi $\mathrm{H}$. Role of duration of catheterization and length of hospital stay on the rate of catheter-related hospitalacquired urinary tract infections. Res Rep Urol. 2015; 7:417. doi: 10.2147/RRU.S75419.

26. Chenoweth C, Saint S. Preventing catheter-associated urinary tract infections in the intensive care unit. Crit Care Clin. 2013;29:19-32. doi: 10.1016/j.ccc.2012.10.005.

27. Boroumand MA, Sam L, Abbasi SH, Salarifar M, Kassaian E, Forghani S. Asymptomatic bacteriuria in type 2 Iranian diabetic women: a cross sectional study. BMC Womens Health. 2006; 6:4. doi: 10.1186/1472-6874-6-4.

28. Makuyana D, Mhlabi D, Chipfupa M, Munyombwe T, Gwanzare L. Asymptomatic bacteriuria among outpatients with diabetes mellitus in an urban black population. Cent Afr J Med. 2002; 48:78-82.

29. Mendoza T, García de los Ríos M, Lafourcade M, Soto C, Durruty P, Alvo M. Asymptomatic bacteriuria in type 2 diabetic women. Rev Med chil. 2002; 130:1001-7.

30. Alberti C, Brun-Buisson C, Burchardi H, Martin C, Goodman S, Artigas A, et al. Epidemiology of sepsis and infection in ICU patients from an international multicenter cohort study. Intensive Care Med. 2002; 28:108-21. doi: 10.1007/s00134-001-1143-z.

Copyright $\odot 2018$ The Author(s); Published by Nickan Research Institute. This is an open-access article distributed under the terms of the Creative Commons Attribution License (http://creativecommons.org/licenses/by/4.0), which permits unrestricted use, distribution, and reproduction in any medium, provided the original work is properly cited. 